\title{
Assistência farmacêutica na atenção primária sob a óptica de prescritores de medicamentos de um município do Seridó Oriental Potiguar
}

\author{
Pharmaceutical Assistance in primary health care from the viewpoint \\ of prescriptors at a county of Eastern Seridó Potiguar
}

Recebido em: 07/08/2020 Aceito em: $23 / 04 / 2021$
Jéssica Soares da ROCHA; Almária Mariz BATISTA Escola Multicampi de Ciências Médicas do Rio Grande do Norte. Universidade Federal do Rio Grande do Norte (EMCM/UFRN). Av. Dr. Carlindo Dantas, 540, $2^{\circ}$ andar, CEP 59300-000. Caicó, RN, Brasil. E-mail:almariamariz@yahoo.com.br

\section{ABSTRACT}

This study aimed to evaluate the perception of prescribers of Primary Health Care drugs in Currais Novos, RN, Brazil about Pharmaceutical Assistance, through a cross-sectional, with quantitative approach study. Data collection took place via an instrument adapted from the National Research on Access, Use, and Rational Use of Medicines methodology. Of the 34 professionals, $41.18 \%$ were physicians and $58.82 \%$ dentists, with a prevalence of females (55.88\%). The prevalent employment contract was the contract (32.35\%) under a 40 hour weekly regime (55.88\%). Most prescribers (73.52\%) knew about the Standardized List of Medicines (73.52\%); however, $61.77 \%$ of these never participated in its elaboration and $61.76 \%$ did not know the procedure to request inclusion and/or exclusion of drugs in the list. Medication protocols and access to the Drug Information Center were not a reality (73.54\% and 2.94\%, respectively). Adoption of the Brazilian Common Denomination was cited by $52.94 \%$ of the respondents. Information on medicines availability at the County pharmacy was referred to as usual (70.59\%), and when the prescribers identified a lack of them, they used to substitute in the prescription (76.47\%). Patients' adherence to the prescription was prevalent for respondents (55.89\%). However, the technical complaints and adverse drug events notification were low (14.71\%). The prescribers' evaluation about pharmaceutical assistance activities presented positive points and others that can be improved. Therefore, it is necessary to bring the prescribers closer to this practice via interprofessional work.

Keywords: primary health care; pharmaceutical services; prescriptions.

\section{RESUMO}

O objetivo do presente estudo foi avaliar a percepção de prescritores de medicamentos da Atenção Primária de Currais Novos, RN, sobre Assistência Farmacêutica. Trata-se de estudo transversal, com abordagem quantitativa. A coleta de dados deu-se via aplicação de instrumento adaptado a partir de metodologia proposta para Pesquisa Nacional sobre Acesso, Utilização e Uso Racional de Medicamentos. Dos 34 profissionais, 
$41,18 \%$ eram médicos e 58,82\% dentistas, com prevalência do sexo feminino (55,88\%). O vínculo empregatício prevalente foi o contrato $(32,35 \%)$ sob regime de $40 \mathrm{~h}$ semanais $(55,88 \%)$. A maioria dos prescritores conhecia a Lista Padronizada de Medicamentos (73,52\%); porém, 61,77\% nunca participaram de sua elaboração e 61,76\% não sabiam como proceder para solicitar inclusão e/ou exclusão de medicamentos nesta. Utilização de protocolos de uso de medicamentos não era uma realidade para $73,54 \%$ dos entrevistados, como também o acesso ao Centro de Informações sobre Medicamentos (2,94\%). A adoção da Denominação Comum Brasileira foi citada como conduta por 52,94\% dos respondentes. Informações sobre disponibilidade de medicamentos na farmácia municipal era comum para 70,59\% dos entrevistados e quando os prescritores identificavam falta deles, substituíam na prescrição $(76,47 \%)$. A adesão dos usuários à prescrição era prevalente para os respondentes (55,89\%), contudo, a notificação de queixas técnicas e eventos adversos a medicamentos era baixa (14,71\%). A avaliação dos prescritores sobre atividades da assistência farmacêutica apresentou pontos positivos e outros passíveis de melhoria, portanto, é necessária maior aproximação dos prescritores em relação a esta prática, via um trabalho interprofissional.

Palavras-chave: atenção primária; assistência farmacêutica; prescrição.

\section{INTRODUÇÃO}

O conceito de atenção primária tem sido repetidamente reinterpretado e redefinido. A Organização Mundial da Saúde (OMS) desenvolveu definição coesa baseada na garantia de que as pessoas tenham acesso a serviços abrangentes de promoção, proteção, prevenção, cura, reabilitação e cuidados paliativos ao longo da vida, ação sistemática sobre os determinantes mais amplos de saúde por meio de políticas públicas e ações baseadas em evidências e participação de indivíduos, famílias e comunidades para otimizar sua saúde, como defensores de políticas que promovam e protejam a saúde, como codesenvolvedores de serviços por meio de sua participação e como cuidadores de saúde de si mesmos e de outras pessoas (1).

Nessa conjuntura, o Ministério da Saúde do Brasil define atenção primária como conjunto de ações de saúde individuais, familiares e coletivas que envolvem promoção, prevenção, proteção, diagnóstico, tratamento, reabilitação, redução de danos, cuidados paliativos e vigilância em saúde, desenvolvidas por meio de cuidado integrado e gestão qualificada, realizada com equipe multiprofissional e dirigida à população em território definido, sobre as quais as equipes assumem responsabilidade sanitária (2).

Nesse contexto, a Política Nacional de Medicamentos define assistência farmacêutica como grupo de atividades relacionadas ao medicamento, destinadas a apoiar ações de saúde demandadas por uma comunidade, envolvendo abastecimento de medicamentos em todas e em cada uma de suas etapas constitutivas, conservação, controle de qualidade, segurança e eficácia terapêutica, acompanhamento e avaliação da utilização, obtenção e difusão de informação sobre medicamentos e educação permanente de profissionais de saúde, paciente e comunidade (3).

Outrossim, a Política Nacional de Assistência Farmacêutica define esta prática como conjunto de ações voltadas a promoção, proteção e recuperação da saúde, tanto individual quanto coletiva, tendo o medicamento como insumo essencial e visando seu acesso e uso racional. Este conjunto envolve pesquisa, desenvolvimento e produção de medicamentos e insumos, bem como sua seleção, programação, aquisição, distribuição, dispensação, garantia da qualidade de produtos e serviços, acompanhamento e avaliação de sua utilização (4).

Desta forma, em consonância com a Política Nacional de Medicamentos, esta apresenta, como pressuposto, garantir medicamentos seguros e eficazes à população, ao menor custo possível e de forma descentralizada. Nesse sentido, destaca-se, dentre outras diretrizes desta Política, a adoção de Relação Nacional de Medicamentos Essenciais (RENAME) (3). A Política Nacional de Atenção Básica também corrobora a utilização da RENAME como estraté- 
gia para acesso e promoção de uso racional de medicamentos em nível de atenção primária (2).

A RENAME abrange medicamentos destinados a tratar problemas de saúde prioritários do ponto de vista de saúde pública, portanto, instrumento estratégico para promoção de uso racional de medicamentos no âmbito do Sistema Único de Saúde (SUS). Resulta de seleção entre medicamentos comparáveis, utilizando-se evidências científicas, visto que o cenário atual do mercado farmacêutico abrange grande arsenal de medicamentos comparáveis quanto a indicação, composição, mecanismo de ação, forma farmacêutica, apresentação, efeitos indesejáveis e eficácia, inviabilizando economicamente o SUS ofertar todos estes produtos a seus usuários $(3,5)$.

Nessa perspectiva, a RENAME é utilizada como instrumento norteador para elaboração das listas estaduais e municipais de medicamentos essenciais, denominadas Relações Estaduais de Medicamentos (RESME) e Relações Municipais de Medicamentos (REMUME), respectivamente. RESME e REMUME são de fundamental importância, visto que estas são elaboradas com respaldo no perfil epidemiológico de cada estado/município, fato que não é abarcado pela RENAME, devido à dimensão continental do Brasil. Diante do exposto, é notório que estes documentos também orientam a prescrição no âmbito do SUS $(5,6)$.

A atualização da RENAME é realizada a cada dois anos, de modo a acompanhar mudanças do perfil de adoecimento geral da população e, assim, ofertar o melhor medicamento. Desde 2011 esta responsabilidade foi atribuída à Comissão Nacional de Incorporação de Tecnologias (CONITEC) no SUS, vinculada ao Ministério da Saúde, abrangendo a incumbência de incorporação, ampliação de uso, exclusão ou alteração de tecnologias em saúde (7).

No entanto, a adesão dos prescritores inseridos no SUS às Listas de Medicamentos Essenciais (LME) ainda é uma problemática em diferentes regiões do país, que pode estar vinculada à cultura de prescrição orientada pela indústria farmacêutica, falta de conhecimento dos medicamentos ofertados pelo SUS ou deficiências na seleção destes medicamentos. O que decorre do fato da não adesão é colocar em risco a saúde do paciente, ao prescre- ver medicamentos novos e com poucas evidências pós-comercialização, gerando impactos na logística de medicamentos e orçamentos de saúde $(5,8)$.

Nesse contexto, para que o acesso da população aos medicamentos seja efetivado, é necessária uma prescrição consciente, priorizando os medicamentos enquadrados nas relações de medicamentos, e o corpo de prescritores do SUS que entenda os conceitos e a importância dos medicamentos essenciais. Isso posto, torna-se necessário averiguar o conhecimento sobre a temática de medicamentos essenciais e RENAME/REMUME, como forma de sensibilizar os profissionais de saúde quanto à importância da adesão as listas de medicamentos essenciais e o acesso dos usuários aos medicamentos.

Logo, este trabalho teve por objetivo avaliar as percepções de médicos e dentistas da atenção primária do município de Currais Novos/RN sobre a assistência farmacêutica municipal.

A cidade de Currais Novos está localizada na microrregião do Seridó Oriental do Rio Grande do Norte, a 345 metros de altitude e a $192 \mathrm{~km}$ da capital do referido estado. Apresenta área territorial de $868,25 \mathrm{~km}^{2}$, população de 44.786 pessoas e densidade demográfica de 49,35 hab./ $\mathrm{km}^{2}$ (9).

A atenção primária deste município conta com 17 Estratégias Saúde da Família (ESF), sendo quatorze na zona urbana e três na zona rural. Há onze Unidades Básicas de Saúde (UBS); três destas, localizadas na zona urbana, contam com duas ESF em atuação, totalizando seis ESF. Algumas ESF são contempladas com os programas Residência Multiprofissional em Atenção Básica e Residência Médica, ambos da Escola Multicampi de Ciências Médicas / Universidade Federal do Rio Grande do Norte (EMCM / UFRN), motivo pelo qual este município foi eleito como local de realização deste estudo.

Com relação à assistência farmacêutica, o município dispõe de uma única farmácia básica, que desenvolve atividades inerentes à Central de Abastecimento Farmacêutico (CAF) e dispensação direta ao usuário, localizada em uma UBS. Logo, toda demanda da área urbana é encaminhada para esta única farmácia e a da zona rural é suprida por kits de medicamentos enviados da CAF às UBS. O quadro de funcionários é composto de apenas um farmacêutico, que desenvolve atividades 
técnico-gerenciais e dispensação de medicamento, e um auxiliar de farmácia.

\section{MÉTODO}

Trata-se de estudo transversal, com abordagem quantitativa. A coleta de dados foi baseada em instrumento adaptado a partir de metodologia proposta para Pesquisa Nacional sobre Acesso, Utilização e Uso Racional de Medicamentos (PNAUM), destinada à avaliação de acesso e uso de medicamentos no Brasil (10).

Os sujeitos do estudo foram médicos e dentistas da atenção primária, tanto do quadro municipal quanto dos programas de Residência Médica e Multiprofissional com atividade na atenção primária do município de Currais Novos, RN, os quais aceitaram participar do estudo mediante assinatura de Termo de Consentimento Livre e Esclarecido (TCLE). Profissionais que se recusaram a participar ou estavam fora do serviço por licença ou afastados por qualquer motivo durante a época da coleta de dados foram excluídos do estudo.
A coleta de dados ocorreu no período de julho de 2019 a abril de 2020, nos locais de trabalho dos entrevistados, com horário previamente agendado, ou via e-mail, quando o entrevistado justificava impossibilidade de horário para participação dentro dos seus turnos de atendimento.

Os dados foram tabulados e analisados via Microsoft Excel (versão 2013). Os dados foram sistematizados e interpretados por meio de análise descritiva mediante frequências absoluta/relativa.

O presente estudo foi aprovado pelo Comitê de Ética e Pesquisa (CEP) da Faculdade de Ciências da Saúde do Trairi (FACISA) / Universidade Federal do Rio Grande do Norte (UFRN), sob CAAE 15008919.0.0000.5568.

\section{RESULTADOS E DISCUSSÃO}

Do universo de 37 profissionais das categorias de interesse do estudo, foram entrevistados 34, dos quais $41,18 \%$ eram médicos e $58,82 \%$ dentistas, prevalecendo o sexo feminino $(55,88 \%)$ (Tabela 1$)$. Três médicos se recusaram a participar da pesquisa.

Tabela 1. Características sociodemográficas dos profissionais de saúde na atenção primária do município de Currais Novos, RN, Brasil (julho de 2019 a abril de 2020)

\begin{tabular}{|c|c|c|c|c|c|}
\hline Variável & Categorias & \multicolumn{2}{|c|}{ Profissional (n) } & \multicolumn{2}{|c|}{ Total } \\
\hline \multirow{2}{*}{ Profissão } & & Médico (a) & Dentista & $\mathrm{n}$ & $\%$ \\
\hline & & 14 & 20 & 34 & 100 \\
\hline \multirow{2}{*}{ Sexo } & Feminino & 4 & 15 & 19 & 55,88 \\
\hline & Masculino & 10 & 5 & 15 & 44,12 \\
\hline \multirow{4}{*}{ Vínculo empregatício } & Concurso & & 8 & 8 & 23,53 \\
\hline & Contrato & 5 & 6 & 11 & 32,35 \\
\hline & Mais Médicos & 5 & & 5 & 14,71 \\
\hline & Residência & 4 & 6 & 10 & 29,41 \\
\hline \multirow{2}{*}{ Carga horária } & 30 horas & 4 & 10 & 14 & 41,18 \\
\hline & 40 horas & 9 & 10 & 19 & 55,88 \\
\hline \multirow{3}{*}{ Tempo de atuação na UBS } & $<1$ ano & 9 & 6 & 15 & 44,12 \\
\hline & $1-5$ anos & 5 & 6 & 11 & 32,35 \\
\hline & $>5$ anos & & 8 & 8 & 23,52 \\
\hline \multirow{2}{*}{ Pós graduação } & Sim & 3 & 8 & 11 & 32,35 \\
\hline & Não & 11 & 12 & 23 & 67,65 \\
\hline
\end{tabular}


O perfil sociodemográfico dos profissionais da saúde alterou-se, ao longo dos anos, de cenário quase exclusivamente masculino para outro de distribuição mais equilibrada entre os gêneros masculino e feminino ou até mesmo casos em que mulheres são maioria de algumas profissões desta área, processo chamado de "feminização da saúde" (11). Logo, a prevalência do sexo feminino neste estudo corrobora a literatura, 83,3\% (12), 78,26\% (13) e 80,7\% (14).

Por outro lado, quando analisados individualmente por categoria, foi observado predomínio de homens na área médica, também constatado por uma pesquisa anterior que apontou este gênero como majoritário, tanto em nível nacional (54,4\%), quanto no Rio Grande do Norte (56,1\%) (15). Este cenário pode estar atrelado à faixa etária mais elevada, visto que o perfil dos médicos mais jovens tende à maior proporção feminina $(15,16)$.

A maioria possuía, como vínculo empregatício, um contrato temporário $(32,35 \%)$ e o quadro médico não dispunha de profissional concursado, pois, para esta categoria, há dificuldades de fixação do profissional no município - mesmo concursado - o que justifica, em parte, prevalência de tempo de atuação no serviço menor que 1 ano (44,12\%). Quanto ao regime de trabalho, prevalecia $40 \mathrm{~h}$ semanais $(55,88 \%)$. Em relação ao nível de instrução, a maioria não possuia pós-graduação $(67,65 \%)$ (Tabela 1), o que pode interferir, em parte, na percepção sobre assistência farmacêutica, considerando que a pós-graduação poderia ser uma oportunidade de apropriação de conhecimentos acerca deste tema.

A contratação temporária promove fragilidades ao SUS, com consequências negativas para a qualidade e a continuidade dos serviços e enfraquecimento da relação equipe-comunidade, essencial à atenção básica; contudo, os dados deste estudo reafirmam os de outros $(63,4 \%)(12)$ e $(57,14 \%)$ (17) para este tipo de vínculo. A baixa permanência dos profissionais na equipe também foi constatada em outros estudos (56,76\%) (18) e (41,3\%) (19), o que pode estar correlacionado com falta de concurso público, programas de residência e Mais Médicos e alta rotatividade inerente às contratações (18). Também predominou uma carga horária semanal de 40 horas em outro estudo (80,7\%) (20), o que pode favorecer o desempenho das atividades das equipes de saúde.

A pós-graduação não foi atributo prevalente entre os prescritores, divergindo da literatura. Estudos realizados com profissionais da ESF em João Pessoa, PB, e Goiânia, GO, identificaram que $77,2 \%$ e $59,8 \%$, respectivamente, possuíam pós-graduação $(12,19)$. Este perfil profissional pode ser sugestivo de uma força produtiva recém-formada ou que ainda está em processo de formação em programas de residência médica/ multiprofissional (15).

Quando questionados se o município adotava Lista Padronizada de Medicamentos (LPM), a maioria dos profissionais afirmou que sim (79,41\%). Os itens subsequentes sobre a LPM municipal foram destinados aos que responderam SIM ao questionamento supracitado (Tabela 2).

A maioria dos prescritores $(73,52 \%)$ conhecia a LPM por meio do consultório $(55,88 \%)$ e concordava que atende parcialmente às necessidades dos usuários $(50 \%)$. Porém, nunca participaram de sua elaboração $(61,77 \%)$ nem sabiam como proceder para solicitar inclusão e/ou exclusão de medicamentos nesta $(61,76 \%)$. Os profissionais que conheciam os procedimentos de inclusão e/ou exclusão $(8,82 \%)$ o fizeram influenciados, principalmente, por experiência clínica $(8,82 \%)$. Quanto à adoção de protocolos de uso de medicamentos, a maioria $(58,82 \%)$ relatou não fazer uso de nenhum protocolo (Tabela 2).

$\mathrm{O}$ alto índice de contato prévio com uma LME corrobora com os dados encontrados pela pesquisa desenvolvida por Karnikowski e cols (2017) que afirmaram que $95,3 \%$ dos seus entrevistados prescritores conheciam previamente a LME. O acesso a LME é prioritário por meio dos consultórios, porém a internet surge como veículo importante para contato com a LME na atual pesquisa, fato esse que não é identificado por estudo anterior, que a enquadra como local de menor acesso (21).

Este panorama sugere uma problemática que pode estar acontecendo com os prescritores: a confusão entre conhecimento da íntegra da LME com a divulgação da disponibilidade semanal do estoque da farmácia, efetuado normalmente via in- 
Tabela 2. Conhecimento sobre Lista Padronizada de Medicamentos (LPM) entre os profissionais de saúde na atenção primária do município de Currais Novos, RN, Brasil (julho de 2019 a abril de 2020)

\begin{tabular}{|c|c|c|c|c|c|}
\hline Variável & Categorias & Profiss & al (n) & & \\
\hline & & Médico (a) & Dentista & $\mathrm{n}$ & $\%$ \\
\hline & Sim & 11 & 16 & 27 & 79,41 \\
\hline $\begin{array}{l}\text { Municipio adota Lista Padronizada de } \\
\text { Medicamentos (LPM) }\end{array}$ & Não & 1 & 1 & 2 & 5,88 \\
\hline & Não sei & 2 & 3 & 5 & 14,71 \\
\hline & Sim & 11 & 14 & 25 & 73,52 \\
\hline Conhece a LPM do município & Não & 0 & 2 & 2 & 5,88 \\
\hline & Não se aplica & 0 & 0 & 0 & 0 \\
\hline & Consultório & 10 & 9 & 19 & 55,88 \\
\hline & Internet & 4 & 6 & 10 & 29,41 \\
\hline Tem acesso à LPM em quais destes lugares & Farmácia & 2 & 8 & 10 & 29,41 \\
\hline (mais de 1 alternativa, se houver) & SMS & 2 & 5 & 7 & 20,59 \\
\hline & Email & 2 & 1 & 3 & 8,82 \\
\hline & Não sei & 0 & 6 & 6 & 17,65 \\
\hline & Sim, totalmente & 1 & 3 & 4 & 11,76 \\
\hline Considera que a LPM no município atende & Sim, parcialmente & 6 & 11 & 17 & 50 \\
\hline às necessidades dos usuários & Não & 4 & 0 & 4 & 11,76 \\
\hline & Não sei & 0 & 0 & 0 & 0 \\
\hline & Sim & 2 & 2 & 4 & 11,76 \\
\hline $\begin{array}{l}\text { á participou do processo de elaboração da } \\
\text { LPM do seu municipio? }\end{array}$ & Não & 9 & 12 & 21 & 61,77 \\
\hline & Não respondeu & 0 & 0 & 0 & 0 \\
\hline 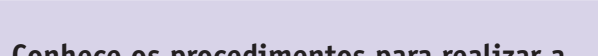 & Sim & 2 & 1 & 3 & 8,82 \\
\hline solicitação de inclusão e/ou exclusão de & Não & 8 & 13 & 21 & 61,76 \\
\hline & Não respondeu & 1 & 0 & 1 & 2,94 \\
\hline & Sim & 2 & 1 & 3 & 8,82 \\
\hline $\begin{array}{l}\text { Já solicitou inclusão/exclusão de } \\
\text { medicamentos na LPM }\end{array}$ & Não & 0 & 0 & 0 & 0 \\
\hline & Não sei & 0 & 0 & 0 & 0 \\
\hline & Artigo científico & 0 & 1 & 1 & 2,94 \\
\hline & Eventos científicos & 0 & 1 & 1 & 2,94 \\
\hline & $\begin{array}{l}\text { Revistas } \\
\text { profissionais }\end{array}$ & 0 & 1 & 1 & 2,94 \\
\hline Essa solicitação foi influenciada por ...? & $\begin{array}{l}\text { Meios de } \\
\text { comunicação }\end{array}$ & 0 & 0 & 0 & 0 \\
\hline & $\begin{array}{l}\text { Representante/ } \\
\text { propagandista }\end{array}$ & 0 & 0 & 0 & 0 \\
\hline & Experiência Clínica & 2 & 1 & 3 & 8,82 \\
\hline
\end{tabular}


ternet (e-mail da UBS); provavelmente alguns se atentaram para essa diferenciação, acarretando na não-conformidade entre os dados de conhecimento de adoção de LME municipal e contato anterior com a LME. Essa circunstância pode ser motivada por falta de divulgação da LME e educação permanente sobre a temática, contato insatisfatório com a assistência farmacêutica e/ou falta de interesse do profissional.

Em referência à LME atender às necessidades dos usuários, os dados confirmam os obtidos por outra pesquisa similar, apontando que 72,8\% (21) dos participantes do Nordeste também se enquadravam na categoria "concordam parcialmente". Todavia, Currais Novos não contava, na ocasião da pesquisa, com uma Comissão de Farmácia e Terapêutica (CFT) formalizada, o que pode implicar em medicamentos inseridos na LME sem os critérios de qualidade, segurança e efetividade estabelecidos pela OMS (21).

A seleção de medicamentos é um processo de escolha baseado em critérios técnicos, epidemiológicos e econômicos realizada por uma CFT e com divulgação dos critérios utilizados para inclusão/exclusão de medicamentos. A CFT possibilita elaboração de listas de medicamentos coesas, com base no perfil de morbimortalidade local e na RENAME, sem presença de medicamentos de utilidade questionável, duplicidade de tratamentos sem significativa vantagem entre eles e inclusão de medicamentos essenciais para algumas terapias especializadas. Estas ações impactam positivamente para assistência farmacêutica e usuários, como otimização da logística da farmácia e redução da exposição de pacientes a fármacos com poucas evidências científicas sobre segurança $(22,23)$.

Na perspectiva econômica, há ganhos significados para o sistema de saúde, como demonstrado por Mato Grosso, que após a criação de sua RESME promoveu uma economia anual de 53,1\%, aproximadamente, R\$ 6.222.196,90 aos cofres públicos ao evitar gastos que resultariam em elevação desnecessária do custo do tratamento, sem necessariamente contribuir com ganhos terapêuticos (24).

A baixa participação dos prescritores no processo de elaboração da LME corrobora estudo brasileiro que constatou que apenas $15 \%$ de seus entrevistados já participaram deste processo e $72,8 \%$ desconhecem como proceder para incluir/ excluir medicamentos. Quanto aos profissionais que conhecem os procedimentos para mudanças na lista, $76,5 \%$ já o fizeram, todavia apenas $54,8 \%$ sob orientação de artigos científicos. Este fato é preocupante, visto que a seleção dos medicamentos deve ser fundamentada em dados científicos e estudos com evidências bem definidas (21).

Um estudo sob responsabilidade da Agência Nacional de Vigilância Sanitária (Anvisa) para investigar a influência da promoção de medicamentos nos processos de assistência farmacêutica dos serviços públicos de saúde do Sistema Único de Saúde (SUS) constatou que 23\% das solicitações de compras de medicamentos não padronizados ou que não constam das listas oficiais podem ser influenciadas pela indústria farmacêutica, $37,7 \%$ dos prescritores alegaram que podem ser influenciados por propagandas e $64,9 \%$ consideram as informações da propaganda para escolher um medicamento no ato da prescrição (25), o que pode justificar, em parte, o constatado em Currais Novos quanto à realização de mudança na LME por prescritores orientada por artigos científicos.

Quanto à presença de protocolos de uso de medicamentos em consultórios, a maioria afirmou que não os possui $(58,82 \%)$ ou não sabe $(14,72 \%)$, totalizando $73,54 \%$ de entrevistados que não utilizam essas ferramentas; contudo, uma outra pesquisa constatou que metade de seus médicos $(49,9 \%)$ (21) possuíam algum formulário terapêutico ou de consensos, diretrizes clínicas ou linhas de cuidado nos consultórios das UBS.

Estes documentos constituem orientação para profissionais de saúde na tomada de decisão, como medicamento a se prescrever, exame diagnóstico solicitar, tempo que o paciente deve ficar internado ou especialidade médica a ser referenciado, resultando em melhora na qualidade do cuidado de saúde ao paciente e orientação adequada na alocação de recursos na assistência à saúde. Algumas possíveis causas da não utilização destas fontes são rigidez na aplicação a pacientes individuais, recomendações muito simplificadas ou "livro de receitas", redução da autonomia médica, desconhecimento sobre determinado protocolo, 
falta de familiaridade, falta de confiança nas evidências que suportam o protocolo ou no órgão que o produziu (26).

Outra fonte de auxílio na tomada de decisão terapêutica é o Centro de Informação sobre Medicamentos (CIM). A maioria dos participantes $(94,12 \%)$ desconhecia este serviço, consequentemente, o número de profissionais que o buscou foi irrisório (2,94\%) (Tabela 3), o que corrobora vários estudos 1,1\% (27), 3,2\% (28) e 7,14\% (29) para categoria médica. O CIM fornece informação criticamente avaliada, contribuindo para promoção de uso racional de medicamentos (30). O Hospital Regional Mariano Coelho, localizado em Currais Novos, RN, instalou este Serviço em 2017 (28), entretanto, mesmo com este cenário positivo, pode estar acontecendo uma falha na divulgação e/ou aproximação deste junto à atenção básica.

A adoção da Denominação Comum Brasileira (DCB) foi citada por pouco mais da metade de nossos entrevistados $(52,94 \%)$ como conduta nas prescrições, o que corrobora outros estudos de avaliação de prescrições na atenção básica no Distrito Federal/DF (76,67\%) (31) e em Caicó/RN (94,3\%) (32). Contudo, ainda está em desacordo com a Lei $\mathrm{n}^{\circ}$ 9.787/99, que determina que todas as prescrições médicas e odontológicas no âmbito do SUS sejam realizadas pela DCB ou, na sua falta, pela Denominação Comum Internacional (DCI) (33).

Dentistas são mais propensos à adoção exclusiva dessas nomenclaturas em suas prescrições, como sugeriu uma pesquisa realizada em Caicó, $\mathrm{RN}$, encontrando $100 \%$ das prescrições aviadas por princípio ativo e em conformidade com REMUNE vigente (34). O uso da DCB/DCI facilita a educação, a informação, aumenta o acesso da população ao medicamento, promove sua utilização racional, reduz confusões entre nomes de medicamentos e facilita obtenção de informações a respeito de fármacos em literatura internacional, pois a denominação comercial varia de país para país (35).

Os prescritores relataram que, geralmente, eram informados sobre a disponibilidade de medicamentos na farmácia municipal (70,59\%) e a maioria considerou que esta informação estava sempre acessível (58,82\%). Logo, quando era identificada falta de medicamentos, a conduta prioritária era analisar a possibilidade de substituição destes na prescrição $(76,47 \%$ ) (Tabela 3). Foi observada concordância com um estudo anterior no que se refere ao conhecimento dos prescritores sobre a disponibilidade dos medicamentos na farmácia municipal $(77,4 \%)(36)$. Esse contato entre a farmácia e esses profissionais sobre a atualização de seu estoque é de suma importância, visto que grande parte dos municípios brasileiros sofrem com a baixa disponibilidade de medicamentos essenciais, comprometendo o controle do problema de saúde e a adesão ao tratamento $(36,37)$.

Quanto à conduta em caso de falta de medicamento no município em estudo, os achados (76,47\%) reafirmam o constatado em um estudo de avaliação em nível nacional, cuja conduta mais prevalente era a verificação da possibilidade de substituição do medicamento prescrito por outro em estoque na farmácia municipal $(77,4 \%)$, estratégia mais viável para tratamento de condições agudas, visto que a substituição de medicamento de uso contínuo pode comprometer o controle da doença ou a adesão à terapia, impactando na efetividade do tratamento (36).

Um estudo desenvolvido em outro município do Seridó Potiguar mostrou que o acesso a medicamentos ocorria, principalmente, via farmácias privadas (65\%) (38), o que sugere prescrição não conforme com a REMUME e/ou não adaptada à disponibilidade da farmácia.

A baixa disponibilidade de medicamentos na atenção primária $(52,9 \%)$ é uma problemática recorrente no setor público, principalmente quando se observa a região Nordeste $(46,3 \%)$ e municípios de pequeno porte, ficando distante dos $80 \%$ de disponibilidade de medicamentos essenciais preconizados pela OMS. Os medicamentos para tratar as Doenças Crônicas Não Transmissíveis são os que mais apresentam faltas, mesmo diante da predominância dessas comorbidades no perfil epidemiológico, com consequentes danos nas taxas morbimortalidade e capacidade resolutiva deste nível de atenção à saúde (36).

Quanto à distribuição de amostras grátis de medicamentos aos usuários, 55,88\% dos respondentes relataram não fazer, porém, quando este item é analisado por categoria profissional, foi constatado que 
Tabela 3. Percepção sobre a Assistência Farmacêutica entre os profissionais de saúde na atenção primária do município de Currais Novos, RN, Brasil (julho de 2019 a abril de 2020)

\begin{tabular}{|c|c|c|c|c|c|}
\hline Variável & Categorias & \multicolumn{2}{|c|}{ Profissionais (n) } & \multicolumn{2}{|c|}{ Total } \\
\hline & & Médico (a) & Dentista & $\mathrm{n}$ & $\%$ \\
\hline \multirow{4}{*}{$\begin{array}{l}\text { Utiliza Denominação Comum Brasileira / } \\
\text { genérica para prescrever? }\end{array}$} & Sim, sempre & 7 & 11 & 18 & 52,94 \\
\hline & Sim, às vezes & 4 & 4 & 8 & 23,53 \\
\hline & Não usa. & 3 & 3 & 6 & 17,65 \\
\hline & Não respondeu & 0 & 2 & 2 & 5,88 \\
\hline \multirow{2}{*}{$\begin{array}{l}\text { Conhece algum Centro de Informações } \\
\text { sobre Medicamentos (CIM)? }\end{array}$} & Sim & 1 & 1 & 2 & 5,88 \\
\hline & Não & 13 & 19 & 32 & 94,12 \\
\hline \multirow{2}{*}{ Já utilizou algum CIM? } & Sim & 0 & 1 & 1 & 2,94 \\
\hline & Não & 1 & 0 & 1 & 2,94 \\
\hline \multirow{3}{*}{$\begin{array}{l}\text { É informado sobre medicamentos } \\
\text { disponíveis na farmácia municipal? }\end{array}$} & Sim & 11 & 13 & 24 & 70,59 \\
\hline & Não & 2 & 6 & 8 & 23,53 \\
\hline & Não respondeu & 1 & 1 & 2 & 5,88 \\
\hline \multirow{3}{*}{$\begin{array}{l}\text { Quanto às informações sobre a } \\
\text { disponibilidade dos medicamentos }\end{array}$} & $\begin{array}{l}\text { Estão sempre } \\
\text { acessíveis }\end{array}$ & 9 & 11 & 20 & 58,82 \\
\hline & $\begin{array}{l}\text { São de forma clara e } \\
\text { objetiva }\end{array}$ & 7 & 9 & 16 & 47,06 \\
\hline & São confiáveis & 4 & 7 & 11 & 32,35 \\
\hline \multirow{4}{*}{$\begin{array}{l}\text { Na falta de medicamento no município, } \\
\text { qual sua conduta? }\end{array}$} & $\begin{array}{l}\text { Recomendo a } \\
\text { compra }\end{array}$ & 8 & 14 & 22 & 64,7 \\
\hline & $\begin{array}{l}\text { Possibilidade de } \\
\text { substituição da } \\
\text { prescrição }\end{array}$ & 12 & 14 & 26 & 76,47 \\
\hline & $\begin{array}{l}\text { Encaminho para a } \\
\text { Farmácia Popular }\end{array}$ & 6 & 10 & 16 & 47,06 \\
\hline & $\begin{array}{l}\text { Encaminho para o } \\
\text { "Aqui tem Farmácia } \\
\text { Popular" }\end{array}$ & 7 & 3 & 10 & 29,41 \\
\hline \multirow{2}{*}{$\begin{array}{l}\text { Disponibiliza amostras grátis de } \\
\text { medicamentos para usuários }\end{array}$} & Sim & 13 & 2 & 15 & 44,12 \\
\hline & Não & 1 & 18 & 19 & 55,88 \\
\hline \multirow{5}{*}{$\begin{array}{l}\text { Em sua opinião, usuários seguem } \\
\text { corretamente a prescrição? }\end{array}$} & Sempre & 0 & 6 & 6 & 17,65 \\
\hline & Repetidamente & 8 & 5 & 13 & 38,24 \\
\hline & Às vezes & 3 & 9 & 12 & 35,29 \\
\hline & Raramente. & 0 & 0 & 0 & 0 \\
\hline & Nunca & 0 & 0 & 0 & 0 \\
\hline \multirow{3}{*}{$\begin{array}{l}\text { Notifica queixas técnicas e eventos } \\
\text { adversos a medicamentos? }\end{array}$} & Sim & 3 & 2 & 5 & 14,71 \\
\hline & Não & 10 & 17 & 27 & 79,41 \\
\hline & Não sei & 1 & 1 & 2 & 5,88 \\
\hline \multirow{3}{*}{$\begin{array}{l}\text { Há alguma interferência política/ } \\
\text { econômica na assistência farmacêutica e/ } \\
\text { ou na dispensação de medicamentos? }\end{array}$} & Sim & 4 & 3 & 7 & 20,59 \\
\hline & Não & 2 & 5 & 7 & 20,59 \\
\hline & Não sei & 8 & 12 & 20 & 58,82 \\
\hline
\end{tabular}


quase a totalidade dos médicos $(92,86 \%)$ o faziam (Tabela 3), o que sugere que os médicos são mais requisitados que os dentistas pelos representantes comerciais da indústria farmacêutica. Além disso, o percentual de distribuição de amostras grátis $(44,12 \%)$ superou o constatado em nível nacional (32,8\%) (39).

A disponibilização de amostras grátis no SUS vai de encontro ao uso racional de medicamento e à lista de medicamentos padronizados. O marketing por meio de amostras grátis visa fidelizar prescritor e usuário, promovendo necessidade de consumo, o que pode resultar em dificuldade de adesão ao tratamento, judicialização da saúde e pressão para padronização do medicamento, mesmo que este não conte com evidência científica (40).

A percepção dos prescritores acerca da adesão dos usuários à prescrição foi um processo que normalmente acontecia $(55,89 \%$ ) (Tabela 3$)$. Todavia, outro estudo realizado em nível nacional mostrou apenas 2,6\% como aderente ao tratamento farmacológico de doenças crônicas (41).

A adesão à terapia é um dos fatores primordiais no contexto do Uso Racional de Medicamentos e a baixa adesão está relacionada, principalmente, a características individuais do paciente, doença em si, medicamentos utilizados e interação entre paciente e serviços de saúde (41). Desfechos clínicos e qualidade de vida de pacientes com doenças crônicas que seguem corretamente a prescrição são significativamente melhores, logo o uso de serviços de saúde em função da exacerbação e/ou complicação de doenças é menor, gerando benefícios econômicos a estados e/ou municípios. Sensibilizar o usuário sobre danos que a doença pode gerar bem como peculiaridades, benefícios e riscos relacionados ao tratamento são estratégias que podem ser desenvolvidas por profissionais da saúde para aumentar adesão ao tratamento (42).

Quanto à notificação de queixas técnicas e eventos adversos a medicamentos, que podem interferir na adesão à prescrição, apenas 14,71\% afirmaram fazê-lo (Tabela 3), o que corrobora um estudo de nível nacional, no qual $12,3 \%$ dos profissionais de saúde haviam realizado ao menos uma notificação (36). As principais causas que levam ao distanciamento da notificação são falta de conhe- cimento sobre a importância de notificar e como fazê-lo, falta de percepção e compreensão dos incidentes, ausência de tradição dos profissionais de saúde em notificar e tempo consumido para preencher a ficha de notificação (43).

A segurança de um medicamento não é absoluta e a notificação de eventos adversos a medicamentos comercializados, realizada por meio do VigiMed, é imprescindível para avaliação da relação benefício-risco dos eventos adversos já conhecidos e dos que possam surgir, podendo resultar em algumas ações, a depender do caso em questão, de publicação de alerta e cartas aos profissionais de saúde, alteração de bula, adoção de medidas cautelares e cancelamento do registro sanitário (44).

A interferência política ou econômica na assistência farmacêutica e/ou na dispensação de medicamentos foi uma temática sobre a qual a maioria preferiu não se posicionar (58,82\%) (Tabela 3$)$, o que pode ter correlação com o tipo de vínculo empregatício, sendo a maioria via contrato, conforme apresentado anteriormente. O vínculo empregatício via contrato está pautado, em sua maioria, em interesses partidários, baseado em interesses eleitorais e questões políticas, podendo vir a interferir em sua atuação profissional, visto que sua autonomia para resolução de problemas no território da equipe fica comprometida (18). Outro estudo similar identificou que seus participantes consideram que há interferência $(38,5 \%)$ na assistência farmacêutica (45).

A aquisição de medicamentos na Atenção Básica é realizada por meio do Componente Básico da Assistência Farmacêutica, cujo financiamento é tripartite (União, Estados e Municípios, sendo R\$ 5,58/hab/ano repassados pela União, R $\$ 2,36 / \mathrm{hab} /$ ano aplicados pelos estados e R $\$ 2,36 /$ hab/ano pelos municípios) e gerenciado pela esfera municipal. Contudo, é observado que alguns municípios aplicam um valor financeiro abaixo do recomendado pela legislação. Podem estar relacionados a este fato, dentre outros, a não aplicação da contrapartida estadual e/ou municipal ou contrapartidas estadual e/ou municipal em desacordo com a pactuação, acarretando em aquisições em quantidades inferiores à necessidade (46), deixando nítida a íntima relação da política e/ou economia com o acesso aos medicamentos pelos usuários. 


\section{CONCLUSÃO}

O perfil dos prescritores da atenção primária do município de Currais Novos, RN, entre 2019 e 2020, era predominantemente do gênero feminino, sem pós-graduação e que utilizava o contrato como vínculo empregatício.

A percepção dos prescritores acerca das atividades da assistência farmacêutica apresentou pontos positivos, como o conhecimento da existência da LME municipal, os medicamentos selecionados para compor a LME e a disponibilidade dos medicamentos na farmácia municipal, e outros passíveis de melhorias, como a participação na construção da LME, conhecimento dos processos de inclusão/ exclusão de medicamentos na LME e notificação de queixas técnicas e eventos adversos a medicamentos, o que pode ser alcançado mediante fortalecimento do trabalho interprofissional.

Depreende-se, a partir dos dados deste estudo, a necessidade de contratação de mais profissionais farmacêuticos, via concurso público, para compor o quadro municipal, visto que são inviáveis o desenvolvimento e a consolidação de todas as atividades técnicos-gerenciais demandadas pela farmácia, além das técnico-assistenciais, por um único profissional farmacêutico, via contrato provisório, conforme constatado à época do estudo.

\section{REFERÊNCIAS}

1. OPAS. Organização Pan-Americana de Saúde. Atenção primária à saúde. Disponível em: https://www.paho.org/ pt/topicos/atencao-primaria-saude. [acessado em: $02 \mathrm{de}$ janeiro de 2021].

2. BRASIL. Ministério da Saúde. Portaria $n^{\circ} 2.436$, de 21 de setembro de 2017. Aprova a Política Nacional de Atenção Básica, estabelecendo a revisão de diretrizes para a organização da Atenção Básica, no âmbito do Sistema Único de Saúde (SUS). Diário Oficial da União, $\mathrm{n}^{\circ}$ 183, 22 de setembro de 2017. Seção 1. p. 68-69.

3. BRASIL. Ministério da Saúde. Portaria $n^{\circ} 3.916$, de 30 de outubro de 1998. Aprova a Política Nacional de Medicamentos. Diário Oficial da União, 10 de novembro de 1998. Seção 1. p. 18.

4. BRASIL. Ministério da Saúde. Resolução $\mathrm{n}^{\circ}$ 338, de 06 de maio de 2004. Aprova a Política Nacional de Assistência Farmacêutica. Diário Oficial da União, 20 de maio de 2004. Seção 1. p. 52.

5. Aziz MM, Calvo MCM, D’Orsi E. Medicamentos prescritos aos idosos em capital do Sul do Brasil e a Relação Municipal de Medicamentos. Cad. Saúde Pública. 2012;28(1):52-64. DOI: 10.1590/S0102-311X 2012000100006.

6. Torres RM, Pepe VLE, Osorio-de-Castro CGS. Estruturação da assistência farmacêutica: plano de ação para a seleção de medicamentos essenciais. Cad. Saúde Colet. 2013;21(2):188-196. DOI: 10.1590/S1414462X2013000200014.

7. BRASIL. Ministério da Saúde. Relação Nacional de Medicamentos Essenciais: RENAME 2017. Brasília: Ministério da Saúde; 2017.

8. Torres RM, Esher A, Caetanos R, Pepe VLE, Osorio-deCastro CGS. Adesão às listas de medicamentos essenciais por médicos brasileiros em atuação no Sistema Único de Saúde. Rev. Bras. Educ. Med. 2014;38(3):323-330. DOI: 10.1590/S0100-55022014000300006.

9. IBGE. Instituto Brasileiro de Geografia e Estatística. [citado em 09 de agosto de 2020]. Disponível em: https:// cidades.ibge.gov.br.

10. BRASIL. Ministério da Saúde. Pesquisa Nacional sobre o Acesso, Utilização e Promoção do Uso Racional de Medicamentos no Brasil - componente avaliação dos serviços de assistência farmacêutica básica: introdução, método e instrumentos. Brasília: Ministério da Saúde; 2016.

11. Moraes LB, Kligerman DC, Cohen SC. Análise do perfil sociodemográfico e do processo de trabalho do cirurgiãodentista inserido no Programa Saúde da Família em três municípios da região serrana do Estado do Rio de Janeiro. Physis Revista de Saúde Coletiva. 2015;25(1):171-186. DOI: 10.1590/S0103-73312015000100010.

12. Brito GEG, Mendes ACG, Santos Neto PM, Farias DN. Perfil dos trabalhadores da Estratégia Saúde da Família de uma capital do Nordeste do Brasil. Rev. APS. 2016;19(3): 434-445.

13. Martins AB, Guarini G, Moreira RC, Pinheiro EFC, Hatisuka MFB, Garcia Junior CS. Perfil sociodemográfico e as ações da equipe de Atenção Básica em saúde do idoso. Braz. J. Surg. Clin. Res. 2018;22(2):7-13.

14. Silveira CGS. Capacidade para o trabalho: análise funcional do profissional da atenção básica. [Dissertação]. Niterói: Universidade Federal Fluminense. 2018.

15. Scheffer M, Cassenote A, Guilloux AGA, Biancarelli A, Miotto BA, Mainardi GM. Demografia Médica no Brasil 2018. São Paulo: FMUSP, CFM, Cremesp; 2018. 
16. Rodrigues ET, Forster AC, Santos LL, Ferreira JBB, Falk JW, Dal Fabbro AL. Perfil e trajetória profissional dos egressos da residência médica em medicina da família e comunidade do estado de São Paulo. Rev. Bras. Educ. Med. 2017 41(4):604-614. DOI: 10.1590/1981-52712015v41n $4 \mathrm{rb} 20160084$.

17. Oliveira Sobrinho N. A precarização do trabalho dos profissionais da Atenção Básica de Saúde de um Distrito Sanitário de Campina Grande/PB. [Dissertação]. Campina Grande: Universidade Estadual da Paraíba. 2017.

18. Barbosa SP, Coelho KA, Carvalho LM, Sarria B, Santos RC, Cavalcante RB. Aspectos que compõem o perfil dos profissionais médicos da Estratégia Saúde da Família: o caso de um município Polo de Minas Gerais. Rev. Bras. Educ. Med. 2019;43(Suppl.1):395-403. DOI: 10.1590/1981-5271v43suplemento1-20180177.

19. Oliveira MPR, Menezes IHCF, Sousa LM, Peixoto MRG. Formação e qualificação de profissionais de saúde: fatores associados à qualidade da Atenção Primária. Rev. Bras. Educ. Med. 2016;40(4):547-559. DOI: $10.1590 / 1981-52712015 \mathrm{v} 40 \mathrm{n} 4 \mathrm{e} 02492014$.

20. Moreira IJB, Horta JA, Duro LN, Borges DT, Cristofari AB, Chaves J, Bassani DCH, Cerizolli ED, Teixeira RM. Perfil sociodemográfico, ocupacional e avaliação das condições de saúde mental dos trabalhadores da Estratégia Saúde da Família em um município do Rio Grande do Sul, RS. Rev Bras Med Fam Comunidade. 2016;11(38):1-12. DOI: 10.5712/rbmfc11(38)967.

21. Karnikowski MGO, Galato D, Meiners MMMA, Silva EV, Gerlack LF, Bós AJG, Leite SN, Álvares J, Guibu IA, Soeiro OM, Costa KS, Costa EA, Guerra Junior AA, Acurcio FA. Caracterização da seleção de medicamentos para a atenção primária no Brasil. Rev Saúde Pública. 2017;51(Suppl 2):1-10. DOI: 10.11606/S15188787.2017051007065.

22. Piacentini LAP. Avaliação da assistência farmacêutica nos municípios pertencentes a 8a Coordenadoria Estadual de Saúde do Rio Grande do Sul. [Especialização]. Porto Alegre: Universidade Federal do Rio Grande do Sul. 2019.

23. Santana RS, Lobo IZF, Cardoso GC, Matos EK, Koga EKCV; Silva WB. Medicamentos e hospitais públicos: o impacto da implantação de Comissões de Farmácia e Terapêutica. Rev. Bras. Farm. Hosp. Serv. Saúde. 2019;9(2):1-6. DOI: 10.30968/rbfhss.2018.092.006.

24. Oliveira HC, Nakata KCF, Marques LD. Impacto econômico como resultado da atuação de uma comissão de farmácia e terapêutica: a experiência de Mato Grosso. J Assist Farmac Farmacoecon. 2019;4(2):10-17. DOI: 10.22563/2525-7323.2019.v4.n2.p.10-17.

25. ANVISA. Agência Nacional de Vigilância Sanitária. Diagnóstico situacional da promoção de medicamentos em unidades de saúde do Sistema Único de Saúde (SUS).
2011. Disponível em: https://antigo.saude.gov.br/images/ pdf/2016/abril/19/3---a--Apresenta----o-reuni--o-CITJulho-2011-links.pdf. [acessado em 02 de janeiro de 2021].

26. Vasconcelos DMM. Adesão dos profissionais de saúde aos protocolos em assistência farmacêutica - medicamentos excepcionais. [Dissertação]. Rio de Janeiro: Escola Nacional de Saúde Pública Sergio Arouca. 2009.

27. Rapkiewicz JC, Trebien HA, Pereira JG, Lacerda RB, Paula CS. Centro de Informação Sobre Medicamentos do Conselho Regional de Farmácia do Paraná (CIM/CRFPR): avaliação do serviço e satisfação do usuário. Rev. Bras. Farm. 2010;91(3):111-118.

28. Martins ABN, Batista AM. Estruturação de Serviço de Informações sobre Medicamentos (SIM) em um hospital do Rio Grande do Norte, Brasil. Infarma - Ciências Farmac. 2019;31(2):121-128. DOI: 10.14450/2318-9312. v31.e2.a2019.pp121-128.

29. Maia AG, Batista AM. Perfil de Solicitações ao Serviço de Informação sobre Medicamentos (SIM) de Um Hospital Materno-Infantil do Seridó Ocidental Potiguar, Brasil. Infarma - Ciências Farmac. 2020;32(2):153-159. DOI: 10.14450/2318-9312.v32.e2.a2020.pp153-159.

30. Sartori AAT, Czermainski SBC. Os centros de informações sobre medicamentos e o acesso e uso racional de medicamentos no Brasil à luz do direito sanitário. RDisan. 2013;13(3):59-89. DOI: 10.11606/ issn.2316-9044.v13i3p59-89.

31. Santos ACS, Volpe CRG, Pinho DLM, Lima LR, Stival MM, Oliveira VD. Erros de prescrição em uma farmácia básica do Distrito Federal. Cienc. Enferm. 2019;25(3): 1-12. DOI: $10.4067 / \mathrm{s} 0717-95532019000100202$.

32. Silva Júnior FC, Batista AM. Prescrições de antimicrobianos em unidades básicas de saúde de um município do Seridó Potiguar, Brasil. Infarma - Ciências Farmac. 2019;31(4):271-276. DOI: 10.14450/2318-9312.v31.e4. a2019.pp271-276.

33. BRASIL. Lei $n^{\circ} 9.787$, de 10 de fevereiro de 1999. Altera a Lei $n^{\circ} 6.360$, de 23 de setembro de 1976, que dispõe sobre a vigilância sanitária, estabelece o medicamento genérico, dispõe sobre a utilização de nomes genéricos em produtos farmacêuticos e dá outras providências. Diário Oficial da União, $\mathrm{n}^{\circ}$ 2, 11 de fevereiro de 1999. Seção 1. p. 1.

34. Batista A, Correia T. Avaliação formal de prescrições odontológicas no âmbito da atenção primária em município do Seridó Potiguar. J Assist Farmac Farmacoecon. 2019;4(2):4-9. DOI: 10.22563/2525-7323.2019.v4.n2. p.4-9.

35. Souza PHR, Oliveira MAS. Indicadores de prescrição médica: uma revisão sistemática. Infarma - Ciências. Farmac. 2015;27(1):5-13. DOI: 10.14450/2318-9312. v27.e1.a2015.pp5-13. 
36. Nascimento RCRM, Álvares J, Guerra Junior AA, Gomes IC, Costa EA, Leite SN, Costa KS, Soeiro OM, Guibi IA, Karnikowski MGO, Acurcio FA. Disponibilidade de medicamentos essenciais na atenção primária do Sistema Único de Saúde. Rev Saúde Pública. 2017;51(Supp12):1-10. DOI: 10.11606/s15188787.2017051007062.

37. Lima CA, Moreira KS, Abreu MHNG, Vieira DMA, Mangueira SAL, Vieira MA, Costa SM. Qualidade do cuidado: avaliação da disponibilidade de insumos, imunobiológicos e medicamentos na Atenção Básica em municípios de Minas Gerais. Rev Bras Med Fam Comunidade. 2019;14(41):1-12. DOI: 10.5712/rbmfc14 (41) 1900 .

38. Bernadino CN, Batista AM. Assistência Farmacêutica na atenção primária à saúde de um município potiguar, Brasil. Infarma - Ciências Farmac. 2019;31(2):86-92. DOI: 10.14450/2318-9312.v31.e2.a2019.pp86-92.

39. Costa EA, Araújo PS, Pereira MT, Souto AC, Souza GS, Guerra Junior AA, Acurcio FA, Guibi IA, Alvares J, Costa KS, Karnikowski MGO, Soeiro OM, Leite SN. Situação sanitária dos medicamentos na atenção básica no Sistema Único de Saúde. Rev Saude Publica. 2017;51(Suppl2):1-12. DOI: 10.11606/S15188787.2017051007106.

40. Boing AC, Zonta R, Manzini F. Promoção do uso racional de medicamentos na atenção básica. Florianópolis: UFSC; 2016.
41. Tavares NUL, Bertoldi AD, Mengue SS, Arrais PSD, Luiza VL, Oliveira MA, Ramos LR, Farias MR, Dal Pizzol TS. Fatores associados à baixa adesão ao tratamento farmacológico de doenças crônicas no Brasil. Rev Saude Publica. 2016;50(Supp12):1-10. DOI: 10.1590/s1518-8787.2016050006150.

42. Araújo NCF, Palhão DMR, Silva VC, Ávila JOL, Cardoso KF, Santos ERF, Lomba FCMS, Carvalho IRA, Souza $\mathrm{BQ}$, Polisel CG. Avaliação da adesão ao tratamento em condições crônicas de saúde por meio do cuidado farmacêutico. Rev. Bras. Farm. Hosp. Serv. Saúde. 2017;8(3):37-41. DOI: 10.30968/rbfhss.2017.083.007.

43. Duarte MD, Batista LM, Albuquerque PMS. Notificação de farmacovigilância em um hospital oncológico sentinela da Paraíba. Rev. Bras. Farm. Hosp. Serv. 2014; 5(1): 7-11.

44. Vogler M. Por que notificar eventos adversos a medicamentos? Bol Farmacoterap. 2019;23(3):5-10.

45. BRASIL. Ministério da Saúde. Pesquisa Nacional sobre o Acesso, Utilização e Promoção do Uso Racional de Medicamentos no Brasil - componente avaliação dos serviços de assistência farmacêutica básica: resultados. Brasília: Ministério da Saúde; 2017.

46. Pontes M, Tavares NUL, Francisco PMSB, Naves JOS. Aplicação de recursos financeiros para a aquisição de medicamentos para a atenção básica em municípios brasileiros. Ciênc. Saúde Coletiva. 2017;22(8):24532462. DOI: $10.1590 / 1413-81232017228.18282016$. 